\title{
Adopting Action Learning in English for an International Trade Course
}

\section{Ho Gun Lee}

\author{
Cheongju University, Cheongju, Korea
}

Objectives: English for International Trade is a practical course focused on the international sale of goods. The author of this paper, who is currently teaching this course, became aware that many students were unable to apply what they learned on site. The purpose of this study is to discuss the introduction of a teaching-learning model in the classroom and to present necessary considerations for doing so.

Methods: Action learning is applied as a teaching-learning model. It would help students improve their collaborative learning and practical application skills by teaming up and solving problems. The lecture time was minimized and much time was spent coaching the students. The students wrote the import and export scenarios and prepared correspondence accordingly. In addition, peer evaluations for the presentations were performed instead of paper exams in order to evaluate learning achievement.

Results: Through this lesson, communication (between professor-students and students-students) and class participation were shown to be improved. As the class progressed, a decrease in the learning achievement gap between students (teams) was also seen. However, students who did not actively participate in spite of the small class size either became passive in class or dropped out. Conclusions: Considering the fact that the international educational is shifting from focusing on lectures to problem solving or maker education and that evaluation methods are shifting from learning achievement evaluations to learning process evaluations, it is necessary to alter the teaching-learning model at the university level.

Key Words: Action Learning, Teaching-Learning Model, Peer Evaluation, Problem Solving Competency, English for International Trade

\section{Introduction}

English for International Trade is a course focused on understanding overseas buyers and sellers, circular letters and pamphlets introducing companies and their products, inquiries of transaction conditions, third party credit inquiries, offers and

Received: Oct 11, 2018 Revised: Oct 25, 2018 Accepted: Nov 3, 2018 Corresponding author: Ho Gun Lee

College of Economics \& Business Administration, Cheongju University, Cheongju 28503, Korea.

Tel: +82-43-229-8192, E-mail: hogun@cju.ac.kr

This is an Open Access article distributed under the terms of the Creative Commons Attribution Non-Commercial License (http://creativecommons.org/licenses/ by-nc/4.0/) which permits unrestricted non-commercial use, distribution, and reproduction in any medium, provided the original work is properly cited.

Copyright $\odot 2019$ Korean Association for Business Communication. acceptances for contracts, agreements on general terms and conditions, agency agreements, and other communication used in the contract process. Additional correspondence include documents that follow the conclusion of a contract, such as those dealing with insurance, shipment, settlement, dispute claims, and replies to these claims. English for International Trade is thus a trade-specific English language course that focuses on communication used in import and export contracts and their implementation.

In international trade, communication incorporates customary expressions based on contract-related terminology. Terminology may hold legal significance and may be composed of special terms related to the terms of the transaction. In such cases, it is important to use idiomatic expressions used by trad- 


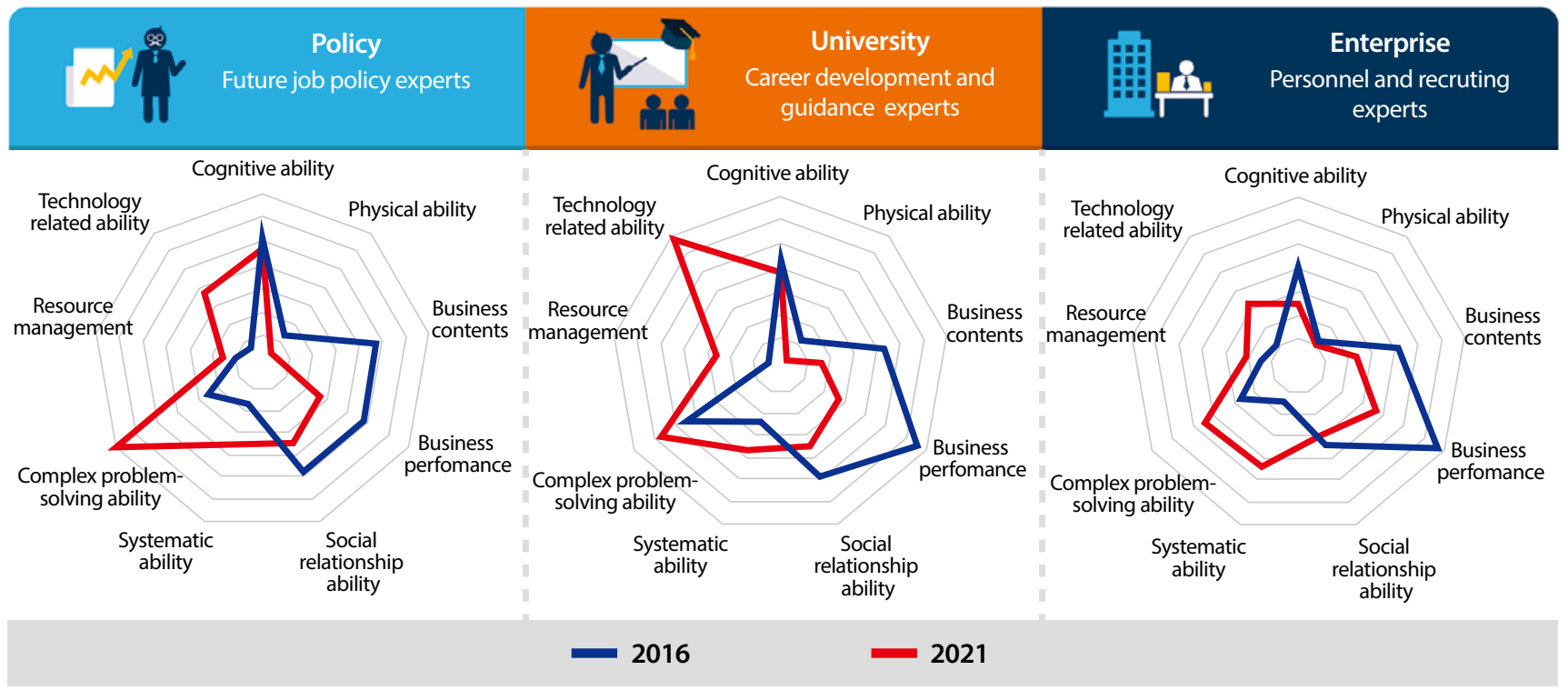

Figure 1. Future core competence (2016 vs. 2021). Adapted from "Korea 10 years later, looking for the way to future job, future strategy report," by Future Preparatory Committee, KISTEP, \& KAIST, 2017, p. 103. Copyright 2017 by Future Preparatory Committee, KISTEP, \& KAIST.

ers for the sake of clarity.

When introducing companies and products, common English expressions are often used. However, regarding credit inquiries, trade terms inquiries, shipment notices, and settlements (e.g., payments, the acceptance and negation of bills of exchange), jargon and limited expressions are often used. In the case of claims and their replies, the way of expressing a statement may vary depending on the given situation, but it is necessary to express it clearly and logically using the correct terms.

The course must thus teach students how to use idiomatic phrases with clear terminology and to construct concise and logical documents and correspondence. Because of the diverse range of goods and situations to be traded, most universities and trade-related educational institutions offer lecture-based classes focusing on correspondence from cases at each stage of the transaction.

\section{Adopting Action Learning}

Traditionally, this course is conducted by explaining various examples of correspondence and idiomatic phrases used in trade. Learners learn how to use the phrases in trade correspondence based on terminology related to cases such as international sales contracts, marine insurance, international logistics, and international payments.

From 2012 to 2018, the author was responsible for the GTEP (Glocal Trade Expert Incubating Program) project supported by MOTIE (Ministry of Trade, Industry and Energy). MOTIE selects 24 universities nationwide, and teams from different universities train and supply advanced trade experts to expand the global market entry of SMEs(Lee, 2015). Each GTEP project team selects approximately 30 students each year with a total of more than 550 hours a year, including 180 hours of regular curriculum, 130 hours of special courses, and 240 hours of on-the-job training. The GTEP project team selects small and medium-sized enterprises wishing to enter the overseas market. They also visit overseas major markets, conduct surveys on overseas markets, participate in overseas exhibitions, and support the export business of companies. When companies participate in overseas expositions, GTEP members participate as field interns or rent exposition booths on behalf of SMEs. On the field, under the supervision of professors, students conduct field sales and negotiation, and upon returning to school, they support trade marketing or trade contracts with the company.

During the GTEP activities, the author became aware of some difficulties students were facing when they were instructed to write to overseas clients on behalf of their companies. They had learned the jargon in other contexts, and they had seen them utilized in their textbooks and on the Internet, but they still found it difficult to write formally and express their opinions in a logical manner. The reason for this is that the students had learned a variety of tools through their lectures but did not have much experience using them in writing. While familiar with idiomatic phrases, they were not familiar with the basic structure of correspondence, and their ability to express ideas logically was weak.

\section{Reasons to Change the Way of Teaching}

As shown in Figure 1, the Future Preparatory Committee con- 
ducted a comparative analysis of the competencies required in 2016 and the competencies required in 2021 for those specializing in future job policies, university guidance and career development, and industry recruiting and personnel.

The competencies presented in the figure can be divided into two categories. On the right side, the main competencies required in 2016 are the knowledge of business content and the ability to improve job performance. On the left side, in 2021, technology-related competencies and complex problem-solving competencies will be required. Currently, education focuses on understanding the content of the work clearly and how to deal with it effectively. In order to prepare for the future, it is necessary to educate students to develop complex problem solving abilities based on their knowledge of technology.

The World Economic Forum (WEF) (2015) analyzed the changes in employment structure in the United States from 1960 to 2009, and the Levy and Murnane (2013) published a report on the changes in employment rates from 1998 to 2009. Comparing these two results, the employment rate of people with advanced problem solving abilities steadily rose due to their ability to maintain interpersonal relationships and think analytically about non-routine problems while the employment rate of routine manual workers or low-level workers fell. In this regard, The WEF (2015), in collaboration with the Boston Consulting Group, presents sixteen technologies in three areas as key competencies that students will need in the future as follows: Foundational Literacies (1. Literacy, 2. Numeracy, 3. Scientific literacy, 4. ICT literacy, 5. Financial literacy, 6. Cultural and civic literacy), Competencies (7. Critical thinking/ problem-solving, 8. Creativity, 9. Communication, 10. Collaboration), Character qualities (11. Curiosity, 12. Initiative, 13. Persistence/grit, 14. Adaptability, 15. Leadership, 16. Social and cultural awareness). In addition, the WEF emphasizes the importance of social-emotional learning.

Lee (2018) summarized the results of the study on the change of learning achievement according to educational method as follows: According to the forgetting curve of Ebbinghaus, forgetting starts from 10 minutes after learning and goes up to about $50 \%$ after 1 hour, $70 \%$ after 1 day, and $80 \%$ after 1 month. According to Bloom's Taxonomy, the lowest level achieved by learners is the memorization of knowledge and goes up according to the steps of comprehension, application, analysis, synthesis, and evaluation. According to Learning Pyramid of NTL (National Training Laboratories), the results of student comprehension increase as follows: $5 \%$ by lecture attendance, going up to $10 \%$ by reading, $20 \%$ by audio-visual education, $30 \%$ by demonstration, $50 \%$ by discussion, $75 \%$ by practice, and $90 \%$ by teaching others. Therefore, even if a learner reaches $30 \%$ learning achievement through good lectures and presentations, they will forget most of what they learned within a month if not they do not acquire it by experience or practice.

Therefore, rather than lecturing on example sentence letters, students were required to write a letter to be used in a trade transaction. In order to enhance their ability to find and solve problems and enable cooperative learning, students teamed up to introduce Action Learning in which students defined import and export items, wrote letters, and evaluated their peers. In summary, the goal of the course was to improve the cooperation and communication between learners and to strengthen the logical cohesion of their communication by applying the content learned together as a group (Chung \& Park, 2015).

\section{Methods}

\section{Outline of Class}

The class participants formed six teams, and each team chose their own team members. Again, the two teams each play a role as a domestic exporter of goods of their choice and as an overseas importer of the opposite team.

Lectures were minimized in order to explain major idioms that are often used in the import and export process. Each team wrote a letter in Korean reflecting the characteristics of the product and their export strategy and then translated it into English. During the class, the professor explained content, composition, and appropriate expressions of the correspondence and then corrected errors in the English sentences.

The learners presented their content as a team. Each team compared and evaluated and ranked the work of the other teams. Each team also performed an internal evaluation performance of each member of their team.

\section{Team Building}

The number of participants was limited to 30 , from which six teams of five were formed. It was necessary to organize the teams evenly in order for the students to make partner teams later. Students were asked to form their own teams to give them a sense of responsibility for their team. First, 6 volunteers who expressed interest in being a team leader were selected, and then each volunteer chose his/her team members one by one.

Each team then selected the commodity to be exported and their target country and was then asked to explain their reasons for selecting their product and target country. After this explanation, the six teams chose a team against which they wished to compete. When a partner team was selected, three groups were formed in each of which two teams were partnered. Each team acted as a domestic exporter for the goods they selected and 
served as an overseas importer for their partner team.

\section{Preparation of Materials}

When a team was formed, the team assigned roles to each member such as overseas export planning, scenario preparation, Korean letter writing, English letter translation, correspondence, and pamphlet design. Later, the evaluation of the members within the team was done on the basis of how well each member performed their role.

Since the two teams became trading partners, they exchanged trade scenarios with each other. The following correspondence and documents were prepared by each team:

- Circular letter and catalog for the company and product: There were no restrictions on the size or number of pages of the catalogs. Ideas and designs that show the features and benefits of the product were favored.

- Trade inquiry, reply, and quotation: Each team was to send a trade inquiry to the other team. The team that received the inquiry replied requesting to implement a transaction and attached a quotation. The quotations were prepared on the basis of the CIF price, taking into consideration domestic sale prices, ordinary production costs, and overseas sale prices.

- Claims and reply: On the assumption that the goods had arrived at the destination, claims were made on the basis of various factors-damaged in transit, shortage of goods, inconsistency of contracted goods, defects of goods, etc. In order to resolve such claims, the claimee was to decide whether to accommodate the claim or refute it on an objective basis and then reply to the claimant.

Preparing the materials preparation and giving the presentation took 3-4 weeks each. In the first week, the professor explained idiomatic phrases for an hour or so. Students selected a topic and exchanged it with each other. The professor used the remaining time of the first week and the second week and one hour of the third week to supervise the students' written materials. At this time, the role of the professor changed from one of a coach to that of a teacher.

\section{Presentation and Evaluation}

At the time of a group's presentation, all materials were to be printed and distributed to the other teams. During the presentation, the other teams evaluated the presenting team. Evaluation scores were given based on a consensus of all the team members.

The main items of evaluation were composed of 5 factors: communication, ability to use media, persuasive power, content composition, and presentation attitude. Presentation time limit was an additional score factor. The evaluation items were scored based on an evaluation table consisting of 7 scales from 14 to 20 points. Accordingly, a minimum score of 70 to a maximum score of 100 was assigned. As the time limit was an additional score factor, the highest score possible was 105 points.

Professors calculated the scores of each team to determine their rank and assigned comprehensive scores to each team (e.g., 100 for the first team and 90 for the second). The team scores that were assigned were apportioned again according to the roles of the team members. The evaluation items were composed of 5 factors as well as team evaluation. The items were evaluated by 7 scales from 14 points to 20 points. The five factors of evaluation were as follows:

- Fidelity to their own role

- Creative opinions in topic selection

- Logical explanations of assertions at the time of discussion

- Level of cooperation and consideration in collaboration

- Meeting initiation and attendance

The team members' scores were apportioned according to the differences of the scores of the team members: 1 st was about $+20 \%$ of the individual average score of the team, 2 nd was about $+10 \%$, $3 \mathrm{rd}$ and 4 th were about the individual average score, 5 th was about $-10 \%$, and 6 th was about $-20 \%$. The final grades were given by adding up the individual points apportioned at the end of each presentation.

\section{Results}

The logical composition of the correspondence, the division of content by paragraphs, the reduction of overlapping expressions, and the composition of concise sentences were all shown to have improved as a result of each team establishing their own topic, practicing the writing of correspondence, and being coached by the professor. At the end of the semester, the differences between the levels of the teams narrowed as the teams tried to improve by studying the correspondence and presentations of the other teams.

Cooperative learning and coaching led to the increase of communication between the professor and students and among students. Competition among the teams led to an increase in student questions even after class. The amount of questions asked was considered to be significant improvement compared to the amount of a typical class.

In order to avoid free riding and scores being too influenced by an individual student, an autonomous effect occurred for the sake of sharing roles. Although there was a difference in level of 
interest and/or ability among each team and member, the overall participation of the class increased by allocating roles to each student.

The disadvantage was that some students failed to adapt to cooperative learning and dropped out of the class. In particular, when one or two students in a team became overbearing, it was likely that the other team members would be neglected. In the case of conflict within a team, the quality of the final output was usually lower. Teams with low enthusiasm were easily distracted while other teams were being coached.

\section{Discussion}

Action learning is an approach to solving real-life problems. It involves taking action and reflecting upon the results. This helps improve the problem-solving process as well as simplify the solutions developed by the team (Revans, 1998; Reynolds, 2011). However, until now, the participants were not familiar with action learning because team activities, evaluation of learning activities, and peer evaluation were not conducted in most of their other classes. In order to solve this problem, it was necessary to explain in detail the contents and significance of team-based learning activities such as role allocation, socialization by communicating with team members, and small leadership in their own role. It was also necessary to explain the learning activities and encourage them to participate in them.

It was essential to provide a clear guide for evaluating the output of other teams for the sake of fair evaluation. The evaluation of the learning process seemed to be done amicably by the evaluation of the team members participation and their role in the learning process. Since there was no objection concerning grades for those two years, it was assumed that the evaluation was considerably objective.

From the viewpoint of professors, in order to introduce such educational methods, it is necessary to establish clear plans for the whole lesson before the class starts. At the same time, students as well as professors should have a better understanding of the teaching-learning model.

The lecture evaluation by students of this class and the evaluation method were extremely divided. The learning effect and student participation in the class had obviously improved, but the class would be regarded as a burden for students who are looking for an easy class. In the case of professors whose lecture evaluation by students is important, it would not be easy to introduce such a teaching and learning method in spite of its high effectiveness.

\section{Conclusion}

No one can simply say which educational method is optimal for a given subject. Furthermore, the introduction of a new teaching-learning model at a university is entirely up to the professor. New methods will be considered a burden by the professor, as they require two to three years to become part of the professor's routine. There is, of course, a possibility that introducing such a method will adversely affect the lecture evaluation by students during that period. Nevertheless, as students can form study teams and address their individual or team projects with their learning coach and at the same time learn the content of project and problem solving process by obtaining knowledge, raising questions, exchanging feedback and introspecting, it is very important to introduce constructive educational methods to the lessons.

\section{References}

Chung, K. H., \& Park, S. G. (2015). The effect of action learning-based teaching and learning strategies on metacognitive, problem solving, interpersonal relationship and team efficacy of nursing students. International Journal of $u$ - and e- Service, Science and Technology, 8(11), 65-74.

Future Preparatory Committee, KISTEP, \& KAIST. (2017). Korea 10 years later, looking for the way to future Job, future strategy report. Goyang, Korea: Jisik Gonggam.

Frey, C. B., \& Osborne, M. A. (2017). The future of employment: How susceptible are jobs to computerisation? Technological Forecasting and Social Change, 114, 254-280.

Lee, H. H. (2015). A study of trade experts incubating in university and the SME export performance. International Commerce and Information Review, 17(2), 307-327.

Lee, H. G. (2018). A study on the confrontation plan of trade education in universities against paradigm shift by the 4 th industrial revolution. The e-Business Studies, 19(1), 187-202.

Revans, R. W. (1998). ABC of action learning. London, UK: Lemos and Crane.

Reynolds, M. (2011). Reflective practice: Origins and interpretations. Action Learning: Research and Practice, 8(1), 5-13.

World Economic Forum (2015). New vision for education: Unlocking the potential of technology. Retrieved from http:// www3.weforum.org/docs/WEFUSA_NewVisionforEducation_ Report2015.pdf 